\title{
Correction to: Hyperkalemia in Chronic Kidney Disease in the New Era of Kidney Protection Therapies
}

\author{
José M. Valdivielso ${ }^{1}$ (D) Olga Balafa ${ }^{2} \cdot$ Robert Ekart $^{3} \cdot$ Charles J. Ferro $^{4} \cdot$ Francesca Mallamaci $^{5} \cdot$ Patrick B. Mark $^{6}$. \\ Patrick Rossignol $^{7} \cdot$ Pantelis Sarafidis $^{8} \cdot$ Lucia Del Vecchio $^{9} \cdot$ Alberto Ortiz $^{10}$
}

Published online: 11 October 2021

(c) Springer Nature Switzerland AG 2021

\section{Correction to: Drugs (2021) 81:1467-1489 https://doi.org/10.1007/s40265-021-01555-5}

The following acknowledgement section should be included in the article:

Acknowledgement: This Review was planned as part of the activity of the European Renal and Cardiovascular Medicine working (EURECAm) group and all authors are EURECAm members.

The original article has been corrected.

The original article can be found online at https://doi.org/10.1007/ s40265-021-01555-5.

José M. Valdivielso

josemanuel.valdivielso@udl.cat

1 Vascular and Renal Translational Research Group, UDETMA, REDinREN del ISCIII, IRBLleida, Lleida, Spain

2 Department of Nephrology, University Hospital of Ioannina, Ioannina, Greece

3 Clinic for Internal Medicine, Department of Dialysis, University Medical Center Maribor, Maribor, Slovenia

4 Department of Renal Medicine, University Hospitals Birmingham, Edgbaston, Birmingham, UK

5 CNR-IFC, Clinical Epidemiology and Pathophysiology of Hypertension and Renal Diseases, Ospedali Riuniti, 89124 Reggio Calabria, Italy
6 Institute of Cardiovascular and Medical Sciences, University of Glasgow, Glasgow, UK

7 Inserm 1433 CIC-P CHRU de Nancy, Inserm U1116 and FCRIN INI-CRCT, Université de Lorraine, Nancy, France

8 Department of Nephrology, Hippokration Hospital, Aristotle University of Thessaloniki, Thessaloníki, Greece

9 Department of Nephrology and Dialysis, Sant'Anna Hospital, ASST Lariana, Como, Italy

10 School of Medicine, IIS-Fundacion Jimenez Diaz, University Autonoma of Madrid, FRIAT and REDINREN, Madrid, Spain 